\title{
A CULTURA DE SEGURANÇA DO PACIENTE NA PERSPECTIVA DO ENFERMEIRO*
}

\author{
Raquel Duarte Corrêa Matiello ${ }^{1}$ Eliane de Fátima Almeida Lima², Maria Carlota Rezende Coelho³, Elizabete \\ Regina Araújo Oliveira ${ }^{4}$, Franciele Marabotti Costa Leite ${ }^{5}$, Cândida Caniçali Primo ${ }^{6}$
}

\begin{abstract}
RESUMO: Estudo transversal com objetivo de descrever as atitudes e cultura de segurança na perspectiva do enfermeiro. Participaram 83 enfermeiros, que responderam ao questionário "Safety Attitudes Questionnaire - Short Form 2006", traduzido para a língua portuguesa. A coleta de dados foi entre abril e maio de 2015 em um hospital localizado em Vitória, Espírito Santo, Brasil. O escore médio total foi de 66, variando de 54,8 para Percepção da gerência a 78,3 para Satisfação no trabalho. Uma percepção positiva da cultura de segurança foi evidenciada por escores $>75$ pontos na média apenas para Satisfação do trabalho. A maioria dos participantes $(89 \%)$ concordam que os erros são tratados de forma apropriada. Concluiu-se que cinco dos seis domínios avaliados tiveram escores negativos, apontando a necessidade de planejamento e desenvolvimento de estratégias voltadas para uma cultura efetiva de segurança dos pacientes.
\end{abstract}

DESCRITORES: Qualidade da assistência à saúde; Segurança do paciente; Avaliação de serviços de saúde; Enfermagem; Cultura organizacional.

\section{PATIENT SAFETY CULTURE FROM THE PERSPECTIVE OF NURSES}

\begin{abstract}
The objective of this cross-sectional study was to describe attitudes and the culture of safety from the perspective of nurses. Eighty-three nurses participated in the study and answered the questionnaire "Safety Attitudes Questionnaire - Short Form 2006", which was translated into Portuguese. Data collection was carried out between April and May 2015 in a hospital located in Vitória, Espírito Santo, Brazil. The mean score was 66, and varied between 54.8 for 'Perception of management' to 78.3 for 'Job satisfaction'. A positive perception of the safety culture was shown by scores of $>75$ points in average only for 'Job satisfaction'. Most participants $(89 \%)$ agree that mistakes are dealt with appropriately. Five of the six fields assessed obtained negative scores, which showed the need for planning and developing strategies oriented toward an effective culture of patient safety. DESCRIPTORS: Quality of health care; Patient safety; Health services evaluation; Nursing; Organizational culture.
\end{abstract}

\section{LA CULTURA DE SEGURIDAD DEL PACIENTE EN LA PERCEPCIÓN DEL ENFERMERO}

RESUMEN: Estudio transversal con el objetivo de describir las actitudes y cultura de seguridad en la perspectiva del enfermero. Participaron 83 enfermeros, que respondieron al cuestionario "Safety Attitudes Questionnaire - Short Form 2006", traducido al portugués. La recolección de datos se efectuó entre abril y mayo de 2015 en un hospital localizado en Vitória, Espírito Santo, Brasil. El puntaje promedio total fue de 66, variando desde 54,8 para Visión de la gerencia a 78,3 para Satisfacción en el trabajo. Se evidenció una percepción positiva a través de puntajes $>75$ puntos promedio solamente en Satisfacción en el trabajo. La mayoría de los participantes $(89 \%)$ concordó en que los errores no son tratados de la manera adecuada. Se concluyó en que cinco de los seis dominios evaluados tuvieron puntajes negativos, expresando la necesidad de planificación y desarrollo de estrategias orientadas a una cultura efectiva de seguridad de los pacientes.

DESCRIPTORES: Calidad de la Atención de Salud; Seguridad del Paciente; Evaluación de Servicios de Salud; Enfermería; Cultura Organizacional.

\footnotetext{
*Artigo extraído da dissertação de mestrado intitulada: "Cultura de segurança do paciente na perspectiva do enfermeiro". UFES, 2015.

${ }^{1}$ Enfermeira. Mestranda em Enfermagem. Secretaria Estadual de Saúde do Espírito Santo. Vitória, ES, Brasil. ${ }^{2}$ Enfermeira. Doutora em Enfermagem. Docente da Universidade Federal do Espírito Santo. Vitória, ES, Brasil.

${ }^{3}$ Enfermeira. Doutora em Enfermagem. Docente da Escola Superior de Ciências, Santa Casa de Misericórdia de Vitória. Vitoria, ES, Brasil.

${ }^{4}$ Enfermeira. Doutora em Enfermagem. Docente da Universidade Federal do Espírito Santo. Vitória, ES, Brasil.

${ }^{5}$ Enfermeira. Doutoranda em Epidemiologia. Docente da Universidade Federal do Espírito Santo. Vitória, ES, Brasil.

${ }^{6}$ Enfermeira. Doutora em Enfermagem. Docente da Universidade Federal do Espírito Santo. Vitória, ES, Brasil.
}

Autor Correspondente:

Raquel Duarte Correa Matiello

Universidade Federal do Espírito Santo

R. Espírito Santense, 16 - 29146-825 - Cariacica, ES, Brasil

E-mail: raquel.matiello123@gmail.com
Recebido: 25/02/2016 Finalizado: $22 / 06 / 2016$ 


\section{INTRODUÇÃO}

Para a Organização Mundial de Saúde (OMS), segurança do paciente corresponde à redução ao mínimo aceitável do risco de dano desnecessário associado ao cuidado de saúde. A Classificação Internacional de Segurança do Paciente define 'segurança do paciente' como o ato de evitar, prevenir ou melhorar os resultados adversos ou as lesões originadas no processo de atendimento médicohospitalar ${ }^{(1)}$. Busca-se instituir a segurança nas organizações de saúde enquanto processo cultural, promovendo maior consciência dos profissionais quanto à cultura de segurança, ao compromisso ético no gerenciamento de risco e com a consequente aquisição de segurança para si e para os usuários atendidos, suprindo a lacuna existente no aspecto da segurança do paciente ${ }^{(2)}$.

No Brasil, seguindo os mesmos objetivos da OMS, por iniciativa da Organização Pan-Americana de Saúde, foi estabelecida a Rede Brasileira de Enfermagem e Segurança do Paciente (REBRAENSP), cujo papel fundamental é disseminar e sedimentar a cultura de segurança do paciente ${ }^{(3)}$. Em julho de 2013, a Agência Nacional de Vigilância Sanitária (ANVISA) instituiu a Resolução da Diretoria Colegiada (RDC) 36, determinando que os serviços de saúde desenvolvessem um Plano de Segurança do Paciente, tendo como princípios norteadores a melhoria contínua dos processos de cuidado e do uso de tecnologias da saúde, a disseminação da cultura de segurança, a articulação e integração dos processos de gestão de risco e a garantia das boas práticas de funcionamento do serviço de saúde ${ }^{(4)}$.

Para melhorar a segurança do paciente, é imprescindível implementar uma política institucional de cultura de segurança, embora esse seja um dos mais difíceis passos para uma instituição. Inicialmente, para que essa mudança ocorra, é essencial o levantamento dos fatores organizacionais que impedem a formação de uma cultura de segurança. Nesse sentido, o clima de segurança da organização fornece informações importantes sobre o estado de segurança de um determinado grupo de trabalho ou da organização como um todo(5).

A cultura de segurança em hospitais tem sido considerada um indicador estrutural básico que facilita as iniciativas para reduzir os riscos e os eventos adversos nesse âmbito ${ }^{(6)}$, e que têm índices alarmantes também em hospitais brasileiros. Estudo Ibero-Americano de eventos adversos realizado em 5 países da América Latina, observou 11.379 pacientes internados em 58 hospitais, estimando-se uma prevalência pontual na ocorrência de eventos adversos em torno de 10,5\%, sendo quase $60 \%$ considerados evitáveis. Essa alta prevalência sugere que a segurança do paciente pode representar uma importante questão de saúde pública ${ }^{(7)}$.

Considerando que o profissional enfermeiro desenvolve a interlocução entre o sistema e o paciente, e encontra-se em posição de destaque, esse profissional deve buscar ações que promovam a segurança do paciente ${ }^{(8)}$. O estudo se justifica dada a atual discussão mundial sobre segurança do paciente, sendo necessário conhecer e compreender os fatores que influenciam em uma cultura organizacional. Diante do exposto, objetivou-se descrever a cultura de segurança do paciente na perspectiva do enfermeiro.

\section{MÉTODO}

Estudo transversal, realizado em um hospital terciário, de caráter filantrópico, no município de Vitória, Espírito Santo, Brasil. Possui um total de 265 leitos e atendimento em diversas especialidades. A população do estudo foram os enfermeiros dessa instituição, sendo os critérios de inclusão: possuir vínculo trabalhista superior a 30 dias e aceitar participar da pesquisa e critérios de exclusão: exercer cargos administrativos em setores de assistência indireta, estar afastado por motivo de férias, licença médica ou durante o período da pesquisa. Aplicando-se esses critérios, obteve-se 83 enfermeiros, abordados durante a jornada de trabalho, em local específico destinado para a pesquisa, após ser divulgado internamente pela instituição.

Para coleta de dados, foi utilizado o Questionário de Atitudes de Segurança (Safety Atitudes Questionaire - SAQ) validado e adaptado culturalmente para a realidade dos hospitais brasileiros ${ }^{(5)}$. Esse instrumento é composto por duas partes: a primeira contém 41 questões envolvendo a percepção sobre segurança do paciente. A segunda parte visa a coletar dados do profissional: cargo exercido, gênero, atuação principal e tempo de atuação. Assim, esse instrumento mensura a percepção dos 
profissionais de saúde por meio de seis domínios: 1 - Clima de Trabalho em Equipe: considerado como a qualidade do relacionamento e a colaboração entre os membros de uma equipe (itens 1 a 6 ); 2 Clima de Segurança: percepção dos profissionais quanto ao comprometimento organizacional para segurança do paciente (itens 7 a 13); 3 - Satisfação no Trabalho: visão positiva do local de trabalho (itens 15 a 19); 4 - Percepção do Estresse: reconhecimento de quanto os fatores estressores podem influenciar na execução do trabalho (itens 20 a 23); 5 - Percepção da Gerência: aprovação das ações da gerência ou administração, tanto da unidade em que o profissional atua, quanto do hospital como um todo (itens 24 a 29); e 6 - Condições de Trabalho: percepção da qualidade do ambiente de trabalho (itens 30 a 33).

O escore final do instrumento varia de 0 a 100, zero corresponde à pior percepção de atitudes de segurança pelos profissionais de saúde e 100 à melhor percepção. São considerados valores positivos quando o total do escore é igual a 75. Quanto à pontuação, foi assim ordenada: A- discorda totalmente vale 0 , B- discorda parcialmente vale 25 , C-neutro vale 50 , D-concorda parcialmente vale 75 , E-concorda totalmente vale 100, e X - não se aplica, com valor 0.

A coleta de dados ocorreu entre abril e maio de 2015. Realizou-se estatística descritiva e aplicouse os testes de Mann-Whitney e Kruskal Wallis para associação entre as variáveis gênero, tempo na especialidade e atuação principal, por meio do programa Social Package Statistical Science (SPSS), versão 20.0.

O estudo foi aprovado pelo Comitê de Ética em Pesquisa do Centro de Ciências da Saúde/UFES sob o registro 999557.

\section{RESULTADOS}

Dos 83 participantes do estudo, verificou-se que $76 \%(n=63)$ eram do gênero feminino, 33\% ( $n=28$ ) apresentaram tempo de atuação maior ou igual a 5 anos na unidade, $74 \%(n=62)$ trabalhavam em área de atenção ao adulto, seguidos de $24 \%(n=20)$ em atenção ao adulto e criança. Além disso, 95\% $(n=78)$ não haviam preenchido o instrumento anteriormente. Na Tabela 1, estão apresentadas as médias, medianas e desvio padrão para cada domínio do instrumento.

Pode-se observar na Tabela 1 que, dos seis domínios do SAQ, a Satisfação no trabalho apresentou média maior que 75 (78,39), sendo considerada avaliação positiva no local de trabalho. Os demais domínios apresentaram avaliação abaixo do esperado para a cultura de segurança. O domínio Percepção de Gerência do Hospital obteve o menor resultado $(54,85)$.

Na Tabela 2, os itens de Clima de trabalho em equipe demonstram que $80 \%(n=67)$ relatam ter o apoio dos outros membros da equipe, $78 \%(n=65)$ fazem perguntas e $75 \%(n=63)$ trabalham em equipe. Quanto ao Clima de segurança, 89\% ( $n=64)$ concordam que os erros são tratados de forma apropriada, e $85 \%$ ( $n=71)$ sabem conduzir as questões de segurança.

Nas Tabelas 2, 3 e 4 são apresentadas as distribuições das respostas dos profissionais por item da escala, agrupados por domínios correspondentes.

Tabela 1 - Análise descritiva dos domínios do Questionário de Atitude de Segurança (SAQ). Vitória, ES, Brasil, 2015

\begin{tabular}{lccc} 
Domínios & Média & Desvio Padrão & Mediana \\
\hline Clima de Trabalho em Equipe & 70,1 & 14,5 & 70,8 \\
\hline Clima de Segurança & 70,7 & 15 & 71,4 \\
\hline Satisfação no Trabalho & 78,3 & 17,9 & 80 \\
\hline Percepção do Estresse & 69,4 & 23,7 & 75 \\
\hline Percepção da Gerência & 54,8 & 18,6 & 52 \\
\hline Condições de Trabalho & 67,7 & 25,2 & 75 \\
\hline Sem Domínio & 64,9 & 16,1 & 68,7 \\
\hline SAQ Geral & 66 & 10,4 & 65,8
\end{tabular}


Tabela 2 - Distribuição das respostas dos profissionais enfermeiros por item nos domínios Clima de Trabalho em Equipe e Clima de Segurança. Vitória, ES, Brasil, 2015

\begin{tabular}{|c|c|c|c|c|}
\hline \multirow{2}{*}{$\begin{array}{l}\text { Domínios } \\
\text { Clima de trabalho em equipe }\end{array}$} & \multicolumn{4}{|c|}{ Respostas } \\
\hline & $\begin{array}{l}\text { Discordo* } \\
\text { N(\%) }\end{array}$ & $\begin{array}{l}\text { Neutro } \\
\text { N(\%) }\end{array}$ & $\begin{array}{c}\text { Concordo* } \\
\mathrm{N}(\%)\end{array}$ & $\begin{array}{l}\mathbf{N A * *} \\
\mathbf{N}(\%)\end{array}$ \\
\hline As sugestões do(a) enfermeiro(a) são bem recebidas nesta área. & $13(15)$ & $10(12)$ & $60(72)$ & 0 \\
\hline $\begin{array}{l}\text { Nesta área, é difícil falar abertamente se eu percebo um problema } \\
\text { com o cuidado ao paciente }\left(\mathrm{R}^{* *}\right) \text {. }\end{array}$ & $26(19)$ & $5(6)$ & $50(60)$ & $2(2)$ \\
\hline Nesta área, as discordâncias são resolvidas de modo apropriado. & $15(17)$ & $12(14)$ & $54(65)$ & $2(2)$ \\
\hline $\begin{array}{l}\text { Eu tenho o apoio que necessito de outros membros da equipe } \\
\text { para cuidar dos pacientes. }\end{array}$ & $9(10)$ & $7(8)$ & $67(80)$ & 0 \\
\hline $\begin{array}{l}\text { É fácil para os profissionais que atuam nesta área fazerem } \\
\text { perguntas quando existe algo que eles não entendem. }\end{array}$ & 11(12) & $6(7)$ & $65(78)$ & $1(1)$ \\
\hline $\begin{array}{l}\text { Os(as) médicos(as) e enfermeiros(as) daqui trabalham juntos } \\
\text { como uma equipe bem coordenada. }\end{array}$ & $14(16)$ & $6(7)$ & $63(75)$ & 0 \\
\hline Clima de Segurança & $\begin{array}{l}\text { Discordo* } \\
\text { N(\%) }\end{array}$ & $\begin{array}{l}\text { Neutro } \\
\text { N(\%) }\end{array}$ & $\begin{array}{c}\text { Concordo* } \\
\text { N(\%) }\end{array}$ & $\begin{array}{l}\mathbf{N A * *} \\
\mathbf{N}(\%)\end{array}$ \\
\hline Eu me sentiria seguro(a) se fosse tratado(a) aqui como paciente. & $8(9)$ & $13(15)$ & $57(68)$ & $5(6)$ \\
\hline Erros são tratados de forma apropriada nesta área. & $10(12)$ & $5(6)$ & $64(89)$ & $4(4)$ \\
\hline $\begin{array}{l}\text { Eu conheço os meios adequados para encaminhar as questões } \\
\text { relacionadas à segurança do paciente. }\end{array}$ & $7(8)$ & $3(3)$ & $71(85)$ & $2(2)$ \\
\hline Eu recebo retorno apropriado sobre meu desempenho. & $31(37)$ & $11(13)$ & $39(46)$ & $2(2)$ \\
\hline Nesta área, é difícil discutir sobre erros $\left(\mathrm{R}^{* * *}\right)$. & $31(37)$ & $12(14)$ & $39(46)$ & $1(1)$ \\
\hline $\begin{array}{l}\text { Sou encorajado(a) por meus colegas a informar qualquer } \\
\text { preocupação que eu possa ter quanto à segurança do paciente. }\end{array}$ & $10(12)$ & $10(12)$ & $62(74)$ & $1(1)$ \\
\hline A cultura nesta área torna fácil aprender com os erros dos outros. & $13(15)$ & $7(8)$ & $60(72)$ & $3(3)$ \\
\hline
\end{tabular}

*Parcial ou totalmente; ${ }^{* *}$ Não se aplica; ${ }^{* * *}$ itens reversos

Em relação à Percepção da gerência, Tabela 3, a maioria concordou que a administração da unidade está fazendo um bom trabalho. Quanto a Percepção do estresse, 87\% ( $n=73)$ concordam que a carga de trabalho excessiva prejudica o desempenho.

Na Tabela 4, 89\% ( $n=74)$ dos profissionais afirmou que gosta de seu trabalho e $86 \%(n=72)$ se orgulha de trabalhar em suas áreas, indicando escores positivos.

Na avaliação da associação entre os domínios e as variáveis da caracterização dos enfermeiros, Tabela 5, verificou-se que foi significativa a associação entre SAQ geral e principal área de atuação ( $p$ $=0,02)$, e entre os domínios Clima organizacional e Tempo de especialidade $(p=0,05)$ e Principal área de atuação $(p=0,01)$. Por fim, também houve associação entre Percepção de gerência e Tempo na especialidade $(p=0,04)$. 
Cogitare Enferm. 2016 v. 21 n. esp: 01-09

Tabela 3 - Distribuição das respostas dos profissionais enfermeiros por item nos domínios Percepção da Gerência e do Estresse. Vitória, ES, Brasil, 2015

\begin{tabular}{|c|c|c|c|c|}
\hline Domínios & & Res & stas & \\
\hline Clima de trabalho em equipe & $\begin{array}{l}\text { Discordo* } \\
\mathbf{N}(\%)\end{array}$ & $\begin{array}{l}\text { Neutro } \\
\mathbf{N}(\%)\end{array}$ & $\begin{array}{l}\text { Concordo* } \\
\mathrm{N}(\%)\end{array}$ & $\begin{array}{l}\text { NA** } \\
\text { N(\%) }\end{array}$ \\
\hline As sugestões do(a) enfermeiro(a) são bem recebidas nesta área. & 13(15) & $10(12)$ & $60(72)$ & 0 \\
\hline $\begin{array}{l}\text { Nesta área, é difícil falar abertamente se eu percebo um problema } \\
\text { com o cuidado ao paciente }\left(\mathbf{R}^{* * *}\right) \text {. }\end{array}$ & $26(19)$ & $5(6)$ & $50(60)$ & $2(2)$ \\
\hline Nesta área, as discordâncias são resolvidas de modo apropriado. & $15(17)$ & $12(14)$ & $54(65)$ & $2(2)$ \\
\hline $\begin{array}{l}\text { Eu tenho o apoio que necessito de outros membros da equipe para } \\
\text { cuidar dos pacientes. }\end{array}$ & $9(10)$ & $7(8)$ & $67(80)$ & 0 \\
\hline $\begin{array}{l}\text { É fácil para os profissionais que atuam nesta área fazerem perguntas } \\
\text { quando existe algo que eles não entendem. }\end{array}$ & 11(12) & $6(7)$ & $65(78)$ & $1(1)$ \\
\hline $\begin{array}{l}\text { Os(as) médicos(as) e enfermeiros(as) daqui trabalham juntos como } \\
\text { uma equipe bem coordenada. }\end{array}$ & 14(16) & $6(7)$ & $63(75)$ & 0 \\
\hline Clima de Segurança & $\begin{array}{c}\text { Discordo* } \\
\mathrm{N}(\%)\end{array}$ & $\begin{array}{l}\text { Neutro } \\
\mathrm{N}(\%)\end{array}$ & $\begin{array}{c}\text { Concordo* } \\
\mathrm{N}(\%)\end{array}$ & $\begin{array}{l}\text { NA** } \\
\text { N(\%) }\end{array}$ \\
\hline Eu me sentiria seguro(a) se fosse tratado(a) aqui como paciente. & $8(9)$ & 13(15) & $57(68)$ & $5(6)$ \\
\hline Erros são tratados de forma apropriada nesta área. & 10(12) & $5(6)$ & $64(89)$ & $4(4)$ \\
\hline $\begin{array}{l}\text { Eu conheço os meios adequados para encaminhar as questões } \\
\text { relacionadas à segurança do paciente. }\end{array}$ & $7(8)$ & $3(3)$ & $71(85)$ & $2(2)$ \\
\hline Eu recebo retorno apropriado sobre meu desempenho. & $31(37)$ & 11(13) & $39(46)$ & $2(2)$ \\
\hline Nesta área, é difícil discutir sobre erros $\left(\mathrm{R}^{* * *}\right)$. & $31(37)$ & $12(14)$ & $39(46)$ & $1(1)$ \\
\hline $\begin{array}{l}\text { Sou encorajado(a) por meus colegas a informar qualquer } \\
\text { preocupação que eu possa ter quanto à segurança do paciente. }\end{array}$ & $10(12)$ & $10(12)$ & $62(74)$ & $1(1)$ \\
\hline A cultura nesta área torna fácil aprender com os erros dos outros. & 13(15) & $7(8)$ & $60(72)$ & $3(3)$ \\
\hline
\end{tabular}

*Parcial ou totalmente; ** Não se aplica

Tabela 4 - Distribuição das respostas dos profissionais enfermeiros por item nos domínios Satisfação no trabalho, Condições de trabalho e itens isolados. Vitória, ES, Brasil, 2015

\begin{tabular}{|c|c|c|c|c|}
\hline Domínios & & Resp & ostas & \\
\hline Satisfação do trabalho & $\begin{array}{c}\text { Discordo* } \\
\mathrm{N}(\%)\end{array}$ & $\begin{array}{l}\text { Neutro } \\
\mathrm{N}(\%)\end{array}$ & $\begin{array}{c}\text { Concordo* } \\
\mathrm{N}(\%)\end{array}$ & $\begin{array}{l}\text { NA** } \\
\mathbf{N}(\%)\end{array}$ \\
\hline Eu gosto do meu trabalho. & $4(4)$ & $5(6)$ & $74(89)$ & 0 \\
\hline Trabalhar aqui é como fazer parte de uma grande família. & 10(12) & 13(15) & $60(72)$ & 0 \\
\hline Este é um bom lugar para trabalhar. & $6(7)$ & $11(13)$ & $66(79)$ & 0 \\
\hline Eu me orgulho de trabalhar nesta área. & $4(4)$ & $7(8)$ & $72(86)$ & 0 \\
\hline O moral nesta área é alto. & 15(18) & $10(12)$ & $53(63)$ & $5(6)$ \\
\hline Condições de trabalho & $\begin{array}{l}\text { Discordo* } \\
\mathrm{N}(\%)\end{array}$ & $\begin{array}{l}\text { Neutro } \\
\mathbf{N}(\%)\end{array}$ & $\begin{array}{c}\text { Concordo* } \\
\mathbf{N}(\%)\end{array}$ & $\begin{array}{l}\text { NA** } \\
\text { N(\%) }\end{array}$ \\
\hline $\begin{array}{l}\text { Este hospital faz um bom trabalho no treinamento de novos } \\
\text { membros da equipe. }\end{array}$ & 26(31) & $5(6)$ & $51(61)$ & 1(1) \\
\hline $\begin{array}{l}\text { Toda informação necessária para decisões diagnósticas e } \\
\text { terapêuticas está disponível rotineiramente para mim. }\end{array}$ & $17(20)$ & $10(12)$ & $56(67)$ & 0 \\
\hline Estagiários da minha profissão são adequadamente supervisionados. & 14(16) & $15(18)$ & $47(56)$ & $7(8)$ \\
\hline Itens isolados & $\begin{array}{c}\text { Discordo* } \\
\mathrm{N}(\%)\end{array}$ & $\begin{array}{l}\text { Neutro } \\
\mathbf{N}(\%)\end{array}$ & $\begin{array}{c}\text { Concordo* } \\
\mathrm{N}(\%)\end{array}$ & $\begin{array}{l}N A^{* *} \\
\mathbf{N}(\%)\end{array}$ \\
\hline Eu vivencio boa colaboração com os(as) enfermeiros(as) nesta área. & $5(6)$ & $8(9)$ & $70(84)$ & 0 \\
\hline Eu vivencio boa colaboração com a equipe medica nesta área. & 11(13) & $15(18)$ & $56(67)$ & $1(1)$ \\
\hline Eu vivencio boa colaboração com farmacêuticos nesta área. & $7(8)$ & $12(14)$ & $63(75)$ & 1(1) \\
\hline $\begin{array}{l}\mathrm{R}^{* * *} \text { Falhas na comunicação que levam a atrasos no atendimento } \\
\text { são comuns. }\end{array}$ & $57(68)$ & $5(6)$ & $19(22)$ & 2(2) \\
\hline
\end{tabular}


Tabela 5 - Associação entre os domínios e as variáveis da caracterização dos enfermeiros. Vitória, ES, Brasil, 2015

\begin{tabular}{lcccc} 
Domínios & \multicolumn{3}{c}{ P-valor } \\
\cline { 2 - 5 } & Gênero* & $\begin{array}{c}\text { Tempo na } \\
\text { Especialidade** }\end{array}$ & $\begin{array}{c}\text { Preenchimento } \\
\text { Anterior** }\end{array}$ & $\begin{array}{c}\text { Atuação } \\
\text { Principal** }\end{array}$ \\
\hline Clima Trabalho em Equipe & 0,17 & 0,66 & 0,25 & 0,13 \\
\hline Clima Segurança & 0,38 & 0,05 & 0,78 & 0,01 \\
\hline Satisfação com Trabalho & 0,89 & 0,26 & 0,99 & 0,46 \\
\hline Percepção do Estresse & 0,61 & 0,89 & 0,41 & 0,64 \\
\hline Percepção da Gerência & 0,41 & 0,04 & 0,8 & 0,07 \\
\hline Condições de Trabalho & 0,67 & 0,48 & 0,71 & 0,36 \\
\hline SAQ Geral & 0,82 & 0,11 & 0,92 & 0,02
\end{tabular}

\section{DISCUSSÃO}

Nota-se que a caracterização dos participantes está em concordância com os dados apresentados pelo COREN-ES, a equipe de enfermagem é predominantemente feminina, sendo composta por $88 \%$ de mulheres ${ }^{(9)}$. Em relação ao tempo de atuação, 58\% $(n=48)$ afirmou ter menos de 10 anos de atuação na unidade. Resultados semelhantes foram encontrados em outro estudo, no qual $82,3 \%$ dos participantes possuíam de 1 a 10 anos de trabalho na instituição ${ }^{(8)}$. A atuação principal se deu na atenção ao adulto (74\%), obtendo valor acima que outra pesquisa nacional ${ }^{(8)}$.

Outros estudos no Brasil assemelham a este, e também apresentaram valores inferiores ao escore mínimo recomendado de 75 na maioria das dimensões ${ }^{(10-11)}$. Pesquisa realizada na região sul brasileira, com 75 participantes ${ }^{(12)}$, corrobora com o resultado de escore positivo somente na dimensão Satisfação do trabalho, no entanto, contrapõe-se a outra pesquisa no Sul do Brasil(13) que em todas as dimensões não atingiu o escore.

Em relação ao domínio Trabalho em equipe, assemelha ao de outro estudo ${ }^{(14)}$ realizado em sete hospitais no estado do RS, evidenciando a existência de liberdade ao falar sobre algo que possa afetar negativamente o paciente. Um aspecto importante dentro da instituição é a cooperação, envolvimento e interação entre os diferentes profissionais envolvidos no processo de cuidar, oportunizando visão global de cada situação(15). Além disso, as respostas apontam que existe respeito entre os profissionais entrevistados, assemelhando-se a estudos brasileiros que obtiveram médias parecidas ${ }^{(15-16)}$. Estudo

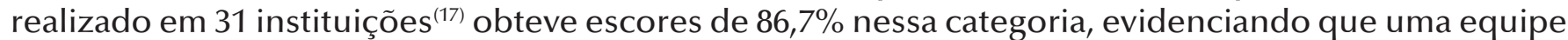
bem relacionada e coordenada propicia uma assistência segura com menores chances de danos ao paciente, visando melhorar o trabalho em equipe como um todo.

A percepção dos profissionais deste estudo sobre o clima de segurança do paciente encontra-se abaixo do esperado corroborando com resultados obtidos em estudo semelhante realizado em seis hospitais da região sul do Brasil( ${ }^{(5)}$ e outro também realizado na mesma região geográfica, com 185 participantes ${ }^{(15)}$. O fato de $46 \%(n=39)$ concordarem que é difícil discutir sobre os erros se contrapõe ao fato de que a maioria dos participantes afirma sentir-se segura sendo tratada como paciente na instituição em que atua, dado que se encontra em concordância com outros estudos (3,18-19). $^{2}$.

A gerência constitui um importante instrumento da política institucional, incorporando um caráter articulador e interativo, sendo assim, o apoio da gestão para a segurança do paciente é uma dimensão no âmbito da organização hospitalar ${ }^{(20)}$, e pode indicar que a visão dos profissionais de enfermagem para a promoção da segurança do paciente não é percebida pela gestão hospitalar, o que foi evidenciado pelo baixo escore obtido nesta pesquisa. A variação das respostas neste domínio reflete a divergência de opiniões dos profissionais no que diz respeito à qualidade do trabalho por ela executado.

Sabe-se que uma das dimensões do processo de cuidado em enfermagem é a dimensão educativa, na qual o enfermeiro é responsável por ensinar os demais membros da equipe. Porém, para proporcionar 
a segurança dentro do ambiente hospitalar, o enfermeiro necessita também receber qualificações dentro dessa área. De acordo com a $\mathrm{OMS}^{(21)}$, é necessário treinar sempre sobre os diversos temas e treinar exaustivamente sobre a segurança do paciente e qualidade do atendimento.

A Percepção do estresse entre os participantes evidencia o entendimento de fatores que comprometem a cultura de segurança. Sabe-se que muitos profissionais estão predispostos a cometer erros pela carga de trabalho excessiva, e tendem a trabalhar descuidados, cometendo erros ${ }^{(12)}$. Além disso, a sobrecarga de trabalho provoca também a insatisfação do profissional em relação à gestão hospitalar ${ }^{(13)}$, fato comprovado neste estudo, em que a percepção da gerência é avaliada de forma negativa. A enfermagem possui uma ferramenta gerencial para realizar a adequação do quantitativo de recursos humanos, respaldada pela legislação do COFEN 293/2004. Porém, a grande maioria das instituições de saúde brasileiras não atinge esse dimensionamento, gerando insuficiência de recursos humanos nos hospitais e riscos para a segurança do paciente ${ }^{(22)}$.

Dessa forma, nessa dimensão, observou-se a grande capacidade do profissional de enfermagem em perceber o quão negativo pode ser o estresse em seu ambiente de trabalho. Essa percepção vinda do enfermeiro está relacionada com seu papel de liderança, visualizando a sobrecarga de trabalho, a fadiga e o estresse como fatores que podem influenciar nas atitudes que podem gerar o erro e comprometer a segurança do paciente ${ }^{(23)}$.

O domínio Satisfação do trabalho foi o único que apresentou valor positivo para a cultura de segurança, resultado semelhante ao encontrado em outro estudo nacional ${ }^{(24)}$ que também aponta a satisfação do profissional, o diálogo e o suporte à equipe por parte dos gestores como fatores essenciais para a segurança do paciente. A satisfação profissional contribui para a maior participação do profissional em seu ambiente de trabalho, tendo em vista que estando mais satisfeitas no trabalho, as pessoas passam a adotar atitudes mais positivas consigo e com os demais colaboradores ${ }^{(25)}$. Destacase o fato de haver uma ligação entre a satisfação no trabalho positiva com o aumento no desempenho e na qualidade na segurança do paciente e consequentemente apoio à criação de uma cultura de segurança ${ }^{(26)}$.

Destaca-se neste estudo a colaboração entre os enfermeiros da mesma área, assemelhando-se a resultados de pesquisa ${ }^{(27)}$ realizada com 130 enfermeiros de todo Brasil, que assinala a importância de um bom relacionamento entre as pessoas com colaboração no trabalho como facilitador para solucionar situações conflituosas. A colaboração e comunicação podem ter implicações para a segurança do paciente, tendo em vista que as falhas na comunicação e na colaboração entre os profissionais de saúde podem constituir fonte potencial de erro, levando à fragmentação dos serviços, bem como a tratamentos e procedimentos técnicos pouco eficazes, com efeitos imediatos e potencialmente negativos para o paciente ${ }^{(28)}$.

\section{CONCLUSÕES}

O instrumento permitiu conhecer a percepção dos enfermeiros acerca da cultura de segurança do paciente na instituição pesquisada, e revelou que cinco dos seis domínios avaliados tiveram escores negativos. O escore médio total foi de 66, variando de 54,8 para Percepção da gerência a 78,3 para Satisfação no trabalho. Uma percepção positiva da cultura de segurança foi evidenciada apenas para Satisfação do trabalho. A maioria dos enfermeiros concordam que os erros são tratados de forma apropriada, referem saber conduzir as questões de segurança, gostam de seu trabalho e orgulham-se de trabalhar em suas áreas, indicando escores positivos.

Aponta-se a necessidade de mudanças em diversos aspectos da cultura de segurança dentro da instituição estudada. Essas mudanças devem ser abrangentes, envolvendo toda a organização do hospital, buscando a adoção da segurança do paciente como uma prioridade estratégica, viabilizando momentos e iniciativas de promoção de práticas seguras.

Como limitação, refere-se que a pesquisa foi realizada apenas com enfermeiros, no entanto, cabe ressaltar que estes representam a maior parte dos trabalhadores que prestam cuidados aos pacientes. Assim, apesar dessa limitação relacionada à categoria profissional, os resultados poderão auxiliar no planejamento e desenvolvimento de estratégias voltadas para uma cultura de segurança dos pacientes 
cada vez mais efetiva.

Considerando que o estudo foi aplicado em uma instituição, sugere-se que outras unidades hospitalares sejam avaliadas, buscando conhecer outras políticas institucionais de segurança do paciente.

\section{REFERÊNCIAS}

1. Agencia Nacional de Vigilância Sanitária (ANVISA). Assistência Segura: Uma Reflexão Teórica Aplicada à Prática. 2013. [acesso em 24 jun 2015]. Disponível:

http://www20.anvisa.gov.br/segurancadopaciente/images/documentos/livros/Livro1-Assistencia_Segura.pdf

2. Claro CM, Krocockz DVC, Toffolleto MC, Padilha KG. Eventos adversos em Unidade de Terapia Intensiva: percepção dos enfermeiros sobre a cultura não punitiva. Rev. esc. enferm USP. [Internet] 2011; 45(1) [acesso em 04 nov 2015] Disponível: http://dx.doi.org/10.1590/S0080-62342011000100023

3. Caldana G, Guirardello EB, Urbanetto JS, Peterlini MAS, Gabriel CS. Rede brasileira de enfermagem e segurança do paciente: desafios e perspectivas Texto contexto - enferm. [Internet] 2015; 24(3) [acesso em 01 fev 2016]. Disponível: http://dx.doi.org/10.1590/0104-070720150001980014

4. Agencia Nacional de Vigilância Sanitária (ANVISA). Resolução da Diretoria Colegiada da ANVISA - RDC n 36, de 25 de Julho de 2013. Institui ações para a segurança do paciente em serviços de saúde e dá outras providências. Diário Oficial da União 2013; 25 Jul.

5. de Carvalho REFL, Cassiani SHB. Questionário Atitudes de Segurança: adaptação transcultural do Safety Attitudes Questionnaire - Short Form 2006 para o Brasil. Rev. Latino-Am. Enfermagem. [Internet] 2012; 20(3) [acesso em 04 nov 2015]. Disponível: http://dx.doi.org/10.1590/S0104-11692012000300020

6. Agency for Healthcare Research and Quality (AHRQ). Making health care safer II: an updated critical analysis of the evidence for patient safety practices. Rockville: Agency for Healthcare Research and Quality; 2013. [acesso em 24 jun 2015]. Disponível: http://www.ahrq.gov/sites/default/files/wysiwyg/research/findings/evidence-basedreports/services/quality/ptsafetyll-full.pdf

7. Aranaz-Andrés JM, Aibar-Rémon C, Limón-Ramirez R, Amarilla A, Restrepo FR, Urroz O, et al. Prevalence of adverse events in the hospitals of five Latin American countries: results of the 'Iberoamerican study of adverse events' (IBEAS). BMJ Qual Saf. [Internet] 2011; 20(12) [acesso em 05 set 2015]. Disponível:

http://dx.doi.org/10.1136/bmjqs.2011.051284

8. da Silva-Batalha SEM, Melleiro MM. Cultura de segurança do paciente em um hospital de ensino: diferenças de percepção existentes nos diferentes cenários dessa instituição. TextoContexto Enferm, Florianópolis. [Internet] 2015; 24(2) [acesso em 20 dez 2015]. Disponível: http://dx.doi.org/10.1590/0104-07072015000192014

9. Fundação Oswaldo Cruz (FIOCRUZ), Conselho Federal de Enfermagem (COFEN), Assossiação Brasileira de Enfermagem (ABEn), Federação Nacional de Enfermeiros (FNE). Perfil da Enfermagem no Brasil. [Internet] 2015. [acesso em 03 dez 2015]. Disponível:

http://www.coren-es.org.br/wp-content/uploads/2015/07/Resultado-Pesquisa-ES.pdf

10. Luiz RB, Simões ALA, Barichello E, Barbosa MH. Fatores associados ao clima de segurança do paciente em um hospital de ensino. Rev. Latino-Am. Enfermagem. [Internet] 2015; 23(5). [acesso em 10 jan 2016]. Disponível: http://dx.doi.org/10.1590/0104-1169.0059.2627

11. Marinho MM, Radunz V, Barbosa SFF. Avaliação da cultura de segurança pelas equipes de enfermagem de unidades cirúrgicas. Texto contexto - enferm. [Internet] 2014; 23(3) [acesso em 05 nov 2015]. Disponível: http://dx.doi.org/10.1590/0104-07072014002640012

12. Misiak M. Cultura de segurança do paciente na perspectiva da equipe de enfermagem de uma instituição cardiovascular [dissertação]. Florianópolis (SC): Universidade Federal de Santa Catarina; 2013.

13. Baratto MAM. Cultura de segurança do paciente: percepções e atitudes dos trabalhadores nas instituições hospitalares de Santa Maria [dissertação]. Rio Grande do Sul (RS): Universidade Federal de Santa Maria; 2015. 
14. Camelo SHH. O trabalho em equipe na instituição hospitalar: uma revisão integrativa. Cogitare enferm. [Internet] 2011; 16(4) [acesso em 22 dez 2015]. Disponível: http://dx.doi.org/10.5380/ce.v16i4.19977

15. Cauduro FLF, Sarquis LM, Sarquis LMM, Cruz EDA. Cultura de segurança entre profissionais de centro cirúrgico. Cogitare enferm. [Internet] 2015; 20(1) [acesso em 06 nov 2015]. Disponível:

http://dx.doi.org/10.5380/ce.v20i1.36645

16. da Correggio TC, Amante LN, Barbosa SFF. Avaliação da cultura de segurança em centro cirúrgico. Rev SOBECC. [Internet] 2014; 19(2) [acesso em 05 nov 2015]. Disponível: http://dx.doi.org/10.4322/sobecc.2014.012

17. Lyu H, Wick CE, Housman M, Freischlag J, Makary MA. A satisfação do paciente como um possível indicador da qualidade da assistência cirúrgica. JAMA Surg. [Internet] 2013; 148(4) [acesso em 05 dez 2015]. Disponível: http://dx.doi.org/10.1001/2013.jamasurg.270

18. Mendes W, Pavao ALB, Martins M, Moura MLO. Características de eventos adversos evitáveis em hospitais do Rio de Janeiro. Rev. Assoc. Med. Bras. [Internet] 2013; 59(5) [acesso em 04 nov 2015]. Disponível:

http://dx.doi.org/10.1016/j.ramb.2013.03.002

19. Reis CT, Laguardia J, Martins M. Adaptação transcultural da versão brasileira do Hospital Surveyon Patient Safety Culture: etapa inicial. Cad. Saúde Pública. [Internet] 2012; 28(11) [acesso em 01 jan 2016]. Disponível: http://dx.doi.org/10.1590/S0102-311X2012001100019

20. de Mello JF, Barbosa SFF. Cultura de segurança do paciente em terapia intensiva: recomendações da enfermagem. Texto contexto - enferm. [Internet] 2013; 22(4) [acesso em 05 nov 2015]. Disponível:

http://dx.doi.org/10.1590/S0104-07072013000400031

21. Organização Mundial da Saúde (OMS). Melhorar a segurança do paciente: primeiros passos. África: Organização Mundial da Saúde, 2012.

22. Versa GLGS, Inoue KC, Nicola AL, Matsuda LM. Influência do dimensionamento da equipe de enfermagem na qualidade do cuidado ao paciente crítico. Texto contexto - enferm. [Internet] 2011; 20(4) [acesso em 20 dez 2015]. Disponível: http://dx.doi.org/10.1590/S0104-07072011000400020

23. Costa DT, Martins MCF. Estresse em profissionais de enfermagem: impacto do conflito no grupo e do poder do médico. Rev. esc. enferm. USP. [Internet] 2011; 45(5) [acesso em 10 dez 2015]. Disponível:

http://dx.doi.org/10.1590/S0080-62342011000500023

24. Rigobello MCG, de Carvalho REFL, Cassiani SHB, Galon T, Capucho HC, de Deus NN. Clima de segurança do paciente: percepção dos profissionais de enfermagem. Acta paul. enferm. [Internet] 2012; (25)5 [acesso em 05 nov 2015]. Disponível: http://dx.doi.org/10.1590/S0103-21002012000500013

25. de Melo MB, Barbosa MA, de Souza PR. Satisfação no trabalho da equipe de enfermagem: revisão integrativa. Rev. Latino-Am. Enfermagem [Internet] 2011; 19(4) [acesso em 06 nov 2015]. Disponível:

http://dx.doi.org/10.1590/S0104-11692011000400026

26. Zakari NMA. Attitude of academic ambulatory nurses toward patient safety culture in Saudi Arabia. Life Scienc J. 2011; 8(3): 230-7.

27. Chaves LD, Ramos LH, de Figueiredo EN. Satisfação profissional de enfermeiros do Trabalho no Brasil. Acta Paul. Enferm. [Internet] 2011; 24(4) [acesso em 01 nov 2015]. Disponível:

http://dx.doi.org/10.1590/S0103-21002011000400010

28. Santos MC, Grilo A, Andrade G, Guimarães T, Gomes A. Comunicação em saúde e a segurança do doente: problemas e desafios. Rev Port Saúde Pública. [Internet] 2010; Temat(10) [acesso em 10 jan 2016]. Disponível: https://www.ensp.unl.pt/dispositivos-de-apoio/cdi/cdi/sector-de-publicacoes/revista/2010/pdf/volumetematico-seguranca-do-doente/6-Comunicacao $\% 20 \mathrm{em} \% 20$ saude $\% 20 \mathrm{e} \% 20 \mathrm{a} \% 20$ seguranca $\% 20 \mathrm{do} \% 20$ doente. pdf 